\title{
LETTER
}

Acute myeloid leukemia

\section{Genomic and evolutionary portraits of disease relapse in acute myeloid leukemia}

 \\ Marc Robert de Massy $\mathbb{D}^{1}$ - Noushin Farnoud ${ }^{1}$. Samuel Haddox ${ }^{4} \cdot$ Tak Lee $^{6}$ - Juan Medina-Martinez ${ }^{1}$. \\ Caroline Sheridan $^{6}$ - Alexis Thurmond ${ }^{4} \cdot$ Michael Becker $^{7}$ - Stefan Bekiranov $\mathbb{B D}^{4} \cdot$ Martin Carroll $^{8}$. \\ Heardly Moses Murdock ${ }^{8} \cdot$ Peter J. M. Valk ${ }^{9} \cdot$ Lars Bullinger $^{10,11} \cdot$ Richard D'Andrea $^{12} \cdot$ Scott W. Lowe ${ }^{5,13}$. \\ Donna Neuberg $\mathbb{( i d}^{14} \cdot$ Ross L. Levine $\mathbb{B}^{1} \cdot$ Ari Melnick $\mathbb{D}^{6} \cdot$ Francine E. Garrett-Bakelman $\mathbb{D}^{4,6,15,16}$
}

Received: 19 October 2020 / Revised: 23 December 2020 / Accepted: 22 January 2021 / Published online: 12 February 2021

(c) The Author(s) 2021. This article is published with open access

\section{To the Editor:}

Relapse in acute myeloid leukemia (AML) patients remains a clinical challenge. The majority of AML patients who receive induction treatment with combination chemotherapy achieve clinicopathologic remission. However, a significant proportion of these patients will relapse and succumb to chemoresistant disease [1]. The biological mechanisms that contribute to relapsed AML are yet to be fully deciphered. Previous studies investigating genetic contributions to AML disease relapse included small numbers of patient samples and/or

These authors contributed equally: Ross L. Levine, Ari Melnick

Supplementary information The online version contains supplementary material available at https://doi.org/10.1038/s41375021-01153-0.

$\triangle$ Francine E. Garrett-Bakelman

fg5q@virginia.edu

1 Molecular Cancer Medicine Service, Human Oncology and Pathogenesis Program, Memorial Sloan Kettering Cancer Center, New York, NY, USA

2 Center for Clinical and Translational Science, The Rockefeller University, New York, NY, USA

3 St. Giles Laboratory of Human Genetics of Infectious Diseases, The Rockefeller University, New York, NY, USA

4 Department of Biochemistry and Molecular Genetics, University of Virginia School of Medicine, Charlottesville, VA, USA

5 Cancer Biology and Genetics Program, Sloan Kettering Institute, Memorial Sloan Kettering Cancer Center, New York, NY, USA

6 Division of Hematology/Oncology, Weill Cornell Medicine, New York, NY, USA

7 Department of Medicine, University of Rochester, Rochester, NY, USA focused on a small number of AML subtypes. These studies have suggested that disease relapse is associated with founder clone recurrence, subclonal expansion and/or the occurrence of relapse-specific events (reviewed in [2]). To better understand the somatic genomic changes that drive AML relapse, we analyzed specimens $(n=120)$ from a clinically annotated adult relapsed AML patient cohort [3] (Supplementary Table S1, Supplementary Fig. S1) for somatic events. The median age of the patient cohort was 50 years. All patients received standard of care combination chemotherapy, achieved complete remission and experienced disease relapse.

We first reanalyzed whole exome sequencing (diagnosis, relapse and matched germlines) of 49 patients [3] in order to capture the complete intragenic mutational burden (Fig. 1A, Supplementary Tables S2 and S3). 21 patients had at least one mutation lost at relapse. Twenty-three patients gained at least one mutation at relapse. A subset of recurrent somatic

8 Division of Hematology and Oncology, University of Pennsylvania Perelman School of Medicine, Philadelphia, PA, USA

9 Department of Hematology, Erasmus MC Cancer Institute, University Medical Center Rotterdam, Rotterdam, the Netherlands

10 Department of Internal Medicine III, University Hospital of Ulm, Ulm, Germany

11 Department of Hematology, Oncology and Tumor Immunology, Charité University Medicine Berlin, Berlin, Germany

12 Centre for Cancer Biology, University of South Australia and SA Pathology, Adelaide, SA, Australia

13 Howard Hughes Medical Institute, Chevy Chase, MD, USA

14 Department of Data Science, Dana Farber Cancer Institute, Boston, MA, USA

15 Department of Medicine, University of Virginia School of Medicine, Charlottesville, VA, USA

16 University of Virginia Cancer Center, Charlottesville, VA, USA 


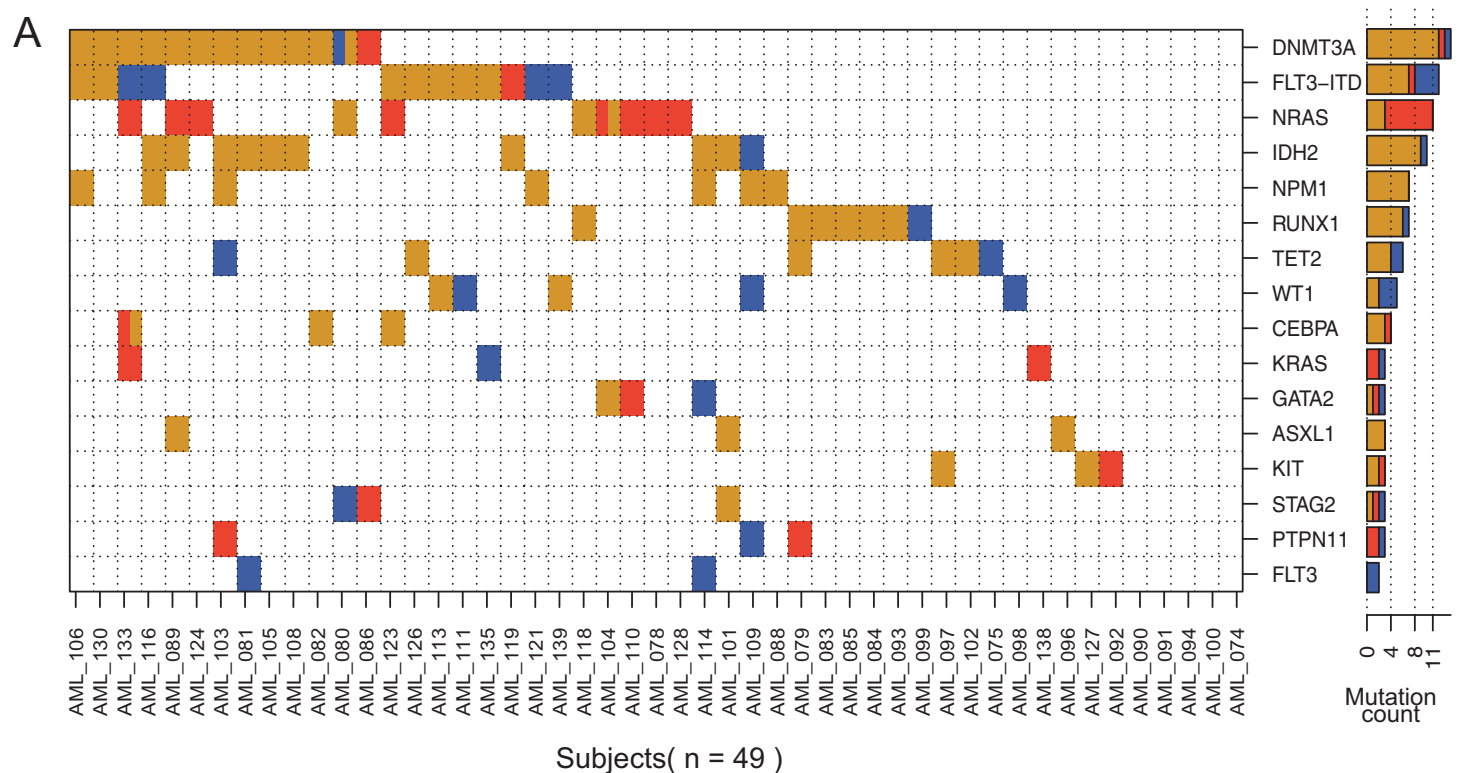

Present at Dx and Rel $\square$ Dx specific $\square$ Rel specific

B

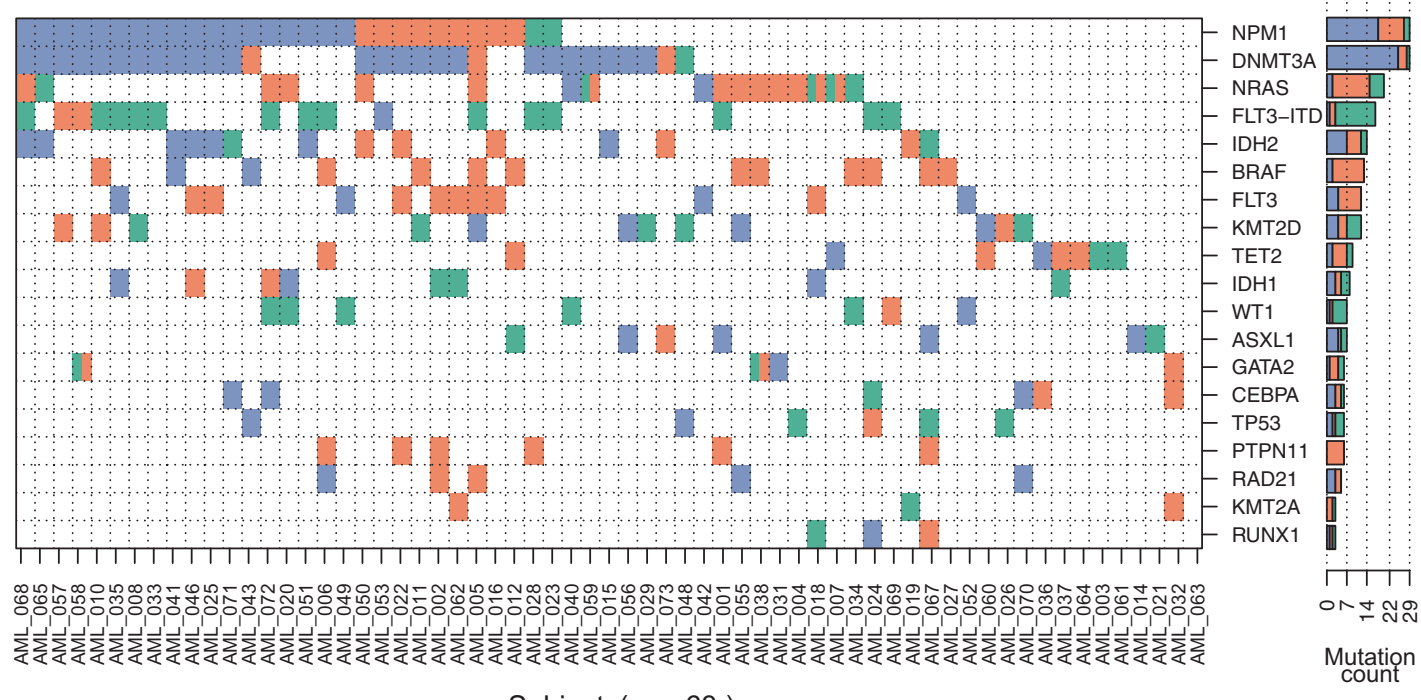

Subjects $(n=63)$

Stable between Dx and Rel

Contracts at Rel

Expands at Rel

Fig. 1 Genomic landscape of relapsed AML. A Comutation map for the whole exome sequencing cohort. Each row is a gene and each column a patient. Mutations were summarized by gene with the exception of FLT3-ITD independently plotted. A cell is colored if the corresponding gene is mutated in the corresponding patient. Every gene that is mutated in at least three patients is included. The bar plot shows the number of patients for which we detected a mutation in this

mutations were validated using orthogonal sequencing (Supplementary Fig. S2; Supplementary Tables S4 and S5). In addition to previously reported commonly mutated genes $[4,5]$, we identified recurrently mutated genes (at least two patients) that were stable or gained upon disease relapse. Other mutations impacted chromatin remodeling (ARIDIB, gene. Colors: brown $=$ events detected in both diagnosis and relapse, red $=$ events only detected at diagnosis, and blue $=$ events only detected at relapse. B Co-mutation map in the targeted panel cohort. Each cell is colored blue if the event is found stable between diagnosis and relapse, orange if it significantly contracts between diagnosis and relapse, and green if it significantly expands between diagnosis and relapse.

BCORL1, CREBBP) and chromatid cohesion (ESPL1) (Supplementary Tables S2 and S3). Previously, mutations in chromatin-related genes at diagnosis were reported to associate with higher rates of relapse [6].

To further understand the patterns of disease progression, we performed copy number alteration (CNA) analyses using 
sparse whole genome sequencing in paired patient specimens ( $n=69$; Supplementary Fig. S3). Results were compared to clinical cytogenetics data and two specimens were removed from the analysis due to discrepant findings. $44.7 \%$ of the 67 patients assessed $(n=30)$ had no detectable CNAs (Supplementary Table S6). In the remaining patients, 34 events were gained and 14 were lost at relapse. A high number of CNAs (three or more unrelated events) was present in $14.9 \%$ of the patients $(n=10)$ : three with CNAs at both diagnosis and relapse, two with diagnosisspecific events, and five with CNAs gained upon relapse. Four of the five cases presented with "atypical" Complex Karyotype disease and were not associated with TP53 mutations [7]. The remaining case exhibited a TP53 R273H mutation that increased in allelic frequency from 0.0864 at diagnosis to 0.281 at relapse with sparse sequencing data revealing associated deletions at $5 \mathrm{q}$ and $17 \mathrm{p}$ among others (Supplementary Fig. S3; Supplementary Tables S2, S3 and S6). These karyotype changes are in agreement with a previous report revealing changes in disease karyotypes upon disease relapse [8].

To identify genetic variation associated with subclone expansion or contraction during disease progression, we implemented a targeted panel sequencing experiment on 63 matched diagnosis and relapse patient specimens. We focused on 38 genes frequently mutated in AML, previously reported as oncogenic and likely-oncogenic somatic events [6] (Supplementary Tables S2b and S7). Genetic variation was considered significantly higher or lower if the difference in allele fraction at relapse compared to diagnosis was at least $0.05 \mathrm{VAF}$ with a significance of $p<0.05$ in a Fisher statistical test (Supplementary Table S7). In more than $50 \%$ of the patients that had a mutation in TP53, WTI or the canonical FLT3-ITD, the mutant subclone expanded at relapse compared to diagnosis (Fig. 1B). By contrast, more than $50 \%$ of the subclones with MAPK activating mutations (e.g., NRAS, PTPN11, and non-ITD FLT3) contracted at relapse (Fig. 1B). Interestingly, in two patients, a sub-clonal NRAS mutation at the time of diagnosis was lost yet they gained another subclonal mutation in the same gene at relapse. Mutations in CEBPA, DNMT3A, and NPMI were more often associated with a clonal fraction that was stable between diagnosis and relapse (Fig. 1B).

We next determined inferred clonal evolution for each patient of the targeted panel sequencing cohort between diagnosis and relapse. Sixty of the patients could be divided into three groups based on the greatest magnitude of change (Fig. 2A; Supplementary Table S8). Group 1: Subclonal changes: 31 patients exhibited significant change(s) in subclonal composition (Supplementary Fig. S4; representative examples in Fig. 2B and Supplementary Fig. S5). Group 2: Clonal changes: 19 patients had either a conversion of at least one subclonal fraction at diagnosis into a clonal event at relapse or a de novo clonal event at relapse (Supplementary Fig. S6; representative example in Fig. 2C. and Supplementary Fig. S7). Group 3: Stable: ten patients had no significant difference observed (Supplementary Fig. S8; representative example in Fig. 2D). In three cases, we could not reconcile the changes between the diagnosis and relapse samples, suggesting either complex dynamics not explained by the models, or the presence of uncommon events outside of the targeted regions.

For three of the patients included in the study, serial specimens were available for further clonal progression assessment (Supplementary Tables S2c and S9). Results were consistent with stable disease after first relapse (AML_124 and AML_126; Supplementary Fig. S9) and the possibility of further subclonal changes during disease progression (AML_130; Fig. 2E). These data further support the occurrence of the proposed evolution models observed throughout the disease time course.

We previously reported that shifts in DNA methylation heterogeneity could classify patients who progress from diagnosis to relapse [3]. We did not find any significant association between the genomic evolution patterns and DNA methylation heterogeneity groupings (Kruskal-Wallis test, $P=0.433$ ). Furthermore, patients' age, sex, ELN classification [9], treatment type, and time to relapse did not significantly associate with the genomic evolution classifications (Kruskal-Wallis test, $P>0.05$; Supplementary Table S10).

Our work suggests that clonal dynamics can potentially contribute to therapeutic resistance and disease progression. Our evolution model predictions are similar to those originally reported from mutational or cytogenetics data [2]. However, we cannot exclude the possibility that alternative drivers of clonal composition were not detected in our data, nor that different treatments will associate with different clonal evolution patterns. Interestingly, the lack of association between epigenetic and genetic evolution progression patterns further supports an independent role for each process during disease progression and the potential for parallel approaches cells can take to disease diversification [3].

Our data suggests that subclonal changes could be pathogenic in the etiology of AML relapse. Expansion of clones with FLT3-ITD at relapse suggests that this enrichment may contribute to disease progression potentially via STAT5 activation, enhanced cell proliferation and/or differentiation blockade [10]. Likewise, expansion of WTI mutations in a subset of patients may contribute to transcriptional dysregulation and impaired hematopoietic differentiation associated with leukemogenesis [11] or to resistance to treatment with DNA damage agents possibly through disrupted TP53 stabilization and transcriptional activity [12]. Finally, our data suggesting the loss of subclones with MAPK activator gene mutations support 

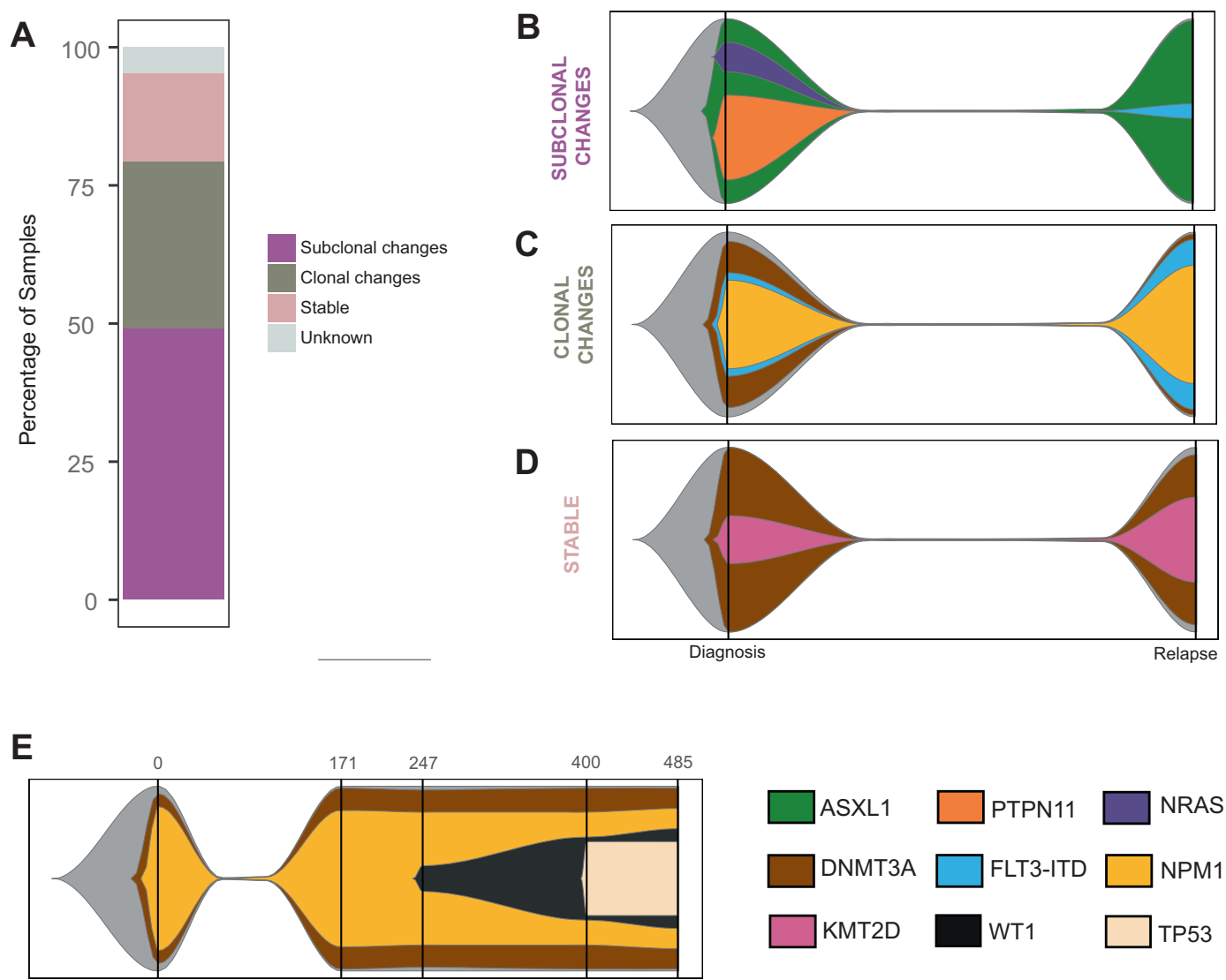

Fig. 2 Clonal evolution during disease progression (targeted panel sequencing cohort). A Partition of the targeted panel sequencing cohort into each of the evolution patterns. Graphical representations of examples of each evolution pattern identified: subclonal (B: AML_001), clonal changes (C: AML_023), and stable changes (D: AML_029) using a fish plot representation. The clone with the highest VAF at a given time point was considered the parent clone.

previous findings consistent with NRAS mutations predisposing leukemic cells to cytarabine-induced differentiation [13]. Changes in FLT3-ITD and karyotype also represent a potential important clinical consideration for treatment of relapsed disease with targeted [14] or PLK1-directed therapy [15]. Importantly, the fact that actionable driver mutations present at diagnosis can be lost or gained at relapse supports a role for temporal monitoring to inform clinicians about possible personalized targeted therapies to consider to maximize clinical benefits in relapsed AML patients.

Acknowledgements The authors acknowledge Elli Papaemmanuil for access to data files, LunBiao Yan (validation targeted resequencing), Nik Cummings, and Andrew Wei (IDH Sequenom) for technical assistance in implementing the respective assays performed.

Funding NCI K08CA169055, UVA Cancer Center through the NCI Cancer Center Support Grant P30 CA44579, the University of Virginia and funding from the American Society of Hematology (ASHAMFDP-20121) under the ASH-AMFDP partnership with The Robert Wood Johnson Foundation and ASH/EHA TRTH to FGB. Partial support UL1 TR001866 from the National Center for
Subclones were defined based on criteria detailed in the Clonal evolution analysis subsection of "Materials and Methods". The color key for gene contributions to the pattern is located in the lower right corner of the figure. E Graphical representation of AML_130 tumor evolution pattern. Each vertical bar indicates a tumor sample collection time point, with the time point (in days) along the $x$-axis.

Advancing Translational Sciences, National Institutes of Health (NIH) Clinical and Translational Science Award (CTSA) program to FR. Starr Cancer Consortium grant I4-A442 to AMM and RL, LLS SCOR 7006-13 to AMM, NCI UG1 CA2333332 to AMM. Funding from the William C. and Joyce C. O'Neil Charitable Trust, Memorial Sloan Kettering Single Cell Sequencing Initiative to TB. NCI grant CA190261 to SWL. The South Australian Cancer Research Biobank (SACRB) is supported by the Cancer Council SA Beat Cancer Project, Medvet Laboratories Pty Ltd and the Government of South Australia. The authors thank the following service providers: Next generation sequencing services were provided by the New York Genome Center and Weill Cornell Medicine Genomics Core facility. Computational resources and technical support were provided by the Weill Cornell Medicine Applied Bioinformatics, the Memorial Sloan Kettering Bioinformatics cores and the School of Medicine Research Computing at The University of Virginia. MRM is currently affiliated with the Cancer Immunology Unit, Research Department of Hematology, University College London Cancer Institute, London, UK, TL is currently currently affiliated with Hunter College, and CS is currently affiliated with Immunai.

Author contributions FEG-B, FR, AMM, and RL conceived the study. Specimen processing and management: TL, CS, SH, and FEGB. Data management and processing: YN, NRF, JM-M, and FEG-B. 
Performed bench experiments and assays: TB, TL, CS, and FEG-B. Performed data analysis: FR, YN, TB, AG, MR, DH, DN, and FEG-B. Provided clinical samples: MB, LB, MC, RDA, and PV Clinical annotation of specimens: AT, LB, and FEG-B. Performed data interpretation and generated figures: FR, YN, and FEG-B. Wrote manuscript: FR and FEG-B. Reviewed results, edited the manuscript and approved final version of the manuscript: all authors.

\section{Compliance with ethical standards}

Conflict of interest The authors declare that they have no conflict of interest.

Publisher's note Springer Nature remains neutral with regard to jurisdictional claims in published maps and institutional affiliations.

Open Access This article is licensed under a Creative Commons Attribution 4.0 International License, which permits use, sharing, adaptation, distribution and reproduction in any medium or format, as long as you give appropriate credit to the original author(s) and the source, provide a link to the Creative Commons license, and indicate if changes were made. The images or other third party material in this article are included in the article's Creative Commons license, unless indicated otherwise in a credit line to the material. If material is not included in the article's Creative Commons license and your intended use is not permitted by statutory regulation or exceeds the permitted use, you will need to obtain permission directly from the copyright holder. To view a copy of this license, visit http://creativecommons. org/licenses/by/4.0/.

\section{References}

1. Szer J. The prevalent predicament of relapsed acute myeloid leukemia. Hematol Am Soc Hematol Educ Program. 2012;2012:43-8.

2. Vosberg S, Greif PA. Clonal evolution of acute myeloid leukemia from diagnosis to relapse. Genes Chromosomes Cancer. 2019;58:839-49.

3. Li S, Garrett-Bakelman FE, Chung SS, Sanders MA, Hricik T, Rapaport F, et al. Distinct evolution and dynamics of epigenetic and genetic heterogeneity in acute myeloid leukemia. Nat Med. 2016;22:792-9.
4. Greif PA, Hartmann L, Vosberg S, Stief SM, Mattes R, Hellmann I, et al. Evolution of cytogenetically normal acute myeloid leukemia during therapy and relapse: an exome sequencing study of 50 patients. Clin Cancer Res. 2018;24:1716-26.

5. Tyner JW, Tognon CE, Bottomly D, Wilmot B, Kurtz SE, Savage SL, et al. Functional genomic landscape of acute myeloid leukaemia. Nature. 2018;562:526-31.

6. Papaemmanuil E, Gerstung M, Bullinger L, Gaidzik VI, Paschka $\mathrm{P}$, Roberts ND, et al. Genomic classification and prognosis in acute myeloid leukemia. N Engl J Med. 2016;374:2209-21.

7. Mrozek K, Eisfeld AK, Kohlschmidt J, Carroll AJ, Walker CJ, Nicolet D, et al. Complex karyotype in de novo acute myeloid leukemia: typical and atypical subtypes differ molecularly and clinically. Leukemia. 2019;33:1620-34.

8. Kern W, Haferlach T, Schnittger S, Ludwig WD, Hiddemann W, Schoch C. Karyotype instability between diagnosis and relapse in 117 patients with acute myeloid leukemia: implications for resistance against therapy. Leukemia. 2002;16:2084-91.

9. Dohner H, Estey E, Grimwade D, Amadori S, Appelbaum FR, Buchner T, et al. Diagnosis and management of AML in adults: 2017 ELN recommendations from an international expert panel. Blood. 2017;129:424-47.

10. Janke H, Pastore F, Schumacher D, Herold T, Hopfner KP, Schneider S, et al. Activating FLT3 mutants show distinct gain-offunction phenotypes in vitro and a characteristic signaling pathway profile associated with prognosis in acute myeloid leukemia. PLoS One. 2014;9:e89560.

11. Rampal R, Alkalin A, Madzo J, Vasanthakumar A, Pronier E, Patel J, et al. DNA hydroxymethylation profiling reveals that WT1 mutations result in loss of TET2 function in acute myeloid leukemia. Cell Rep. 2014;9:1841-55.

12. Bordin F, Piovan E, Masiero E, Ambesi-Impiombato A, Minuzzo S, Bertorelle R, et al. WT1 loss attenuates the TP53-induced DNA damage response in T-cell acute lymphoblastic leukemia. Haematologica. 2018;103:266-77.

13. Brendel C, Teichler S, Millahn A, Stiewe T, Krause M, Stabla K, et al. Oncogenic NRAS primes primary acute myeloid leukemia cells for differentiation. PLoS One. 2015;10:e0123181.

14. Short NJ, Kantarjian H, Ravandi F, Daver N. Emerging treatment paradigms with FLT3 inhibitors in acute myeloid leukemia. Ther Adv Hematol. 2019;10:2040620719827310.

15. Moison C, Lavallee VP, Thiollier C, Lehnertz B, Boivin I, Mayotte N, et al. Complex karyotype AML displays G2/M signature and hypersensitivity to PLK1 inhibition. Blood Adv. 2019;3:552-63. 